\section{Viel Eiweiß oder viele Kohlenhydrate zur Gewichtsreduktion?}

\author{
Eine Gewichtsabnahme ist unabhängig vom Verhältnis der Makro- \\ nährstoffe Eiweiß, Fett und Kohlenhydrate. Es bleibt jedoch die Frage, \\ ob andere Parameter eine andere Sicht nahelegen.
}

n einer randomisierten Studie wurden Körperzusammensetzung Parameter zum oxidativen Stress, zur Proinflammation und zur Betazellfunktion sowie weitere kardiovaskuläre Risikofaktoren untersucht. Das Kollektiv bestand aus 24 adipösen prämenopausalen Frauen mit einem Body Mass Index (BMI) zwischen 30 und $55 \mathrm{~kg} / \mathrm{m}^{2}$. Die Frauen hatten keine kardiovaskulären Risikofaktoren, keinen Diabetes und keine veränderte Nierenfunktion. Sechs Monate lang erhielten sie eine Reduktionskost mit einem Energiedefizit von $500 \mathrm{kcal} / \mathrm{d}$ entweder in Form einer eiweißreichen Kost (EK: 30\% Eiweiß, 30\% Fett, 40\% Kohlenhydrate) oder einer kohlenhydratreichen Kost (KK: 55\% Kohlenhydrate, 30\% Fett, 15\% Eiweiß). Um die Energieaufnahme zu standardisieren, wurden die Nahrungsmittel in frisch verpackter oder gefrorener Form den Probanden ausgehändigt. Die Compliance lag über $90 \%$. Durchgeführt wurden Laboranalysen, eine Glukosebelastung mit Oligosacchariden und einer gemischten Kost sowie eine Dual-Röntgen-Absorptiometrie (DEXA) zur Körperzusammensetzung.

Die Gewichtsabnahme nach sechs Monaten war in beiden Gruppen mit 9,8 und 9,3\% vergleichbar. Im Vergleich zu Frauen mit einer KK verloren Frauen mit einer EK 2,0 kg statt 2,7 kg Muskelmasse, ihr Ruheenergieverbrauch nahm um 16\% zu. Auch die Insulinsensitivität und die Betazellfunktion besserten sich unter einer EK ausgeprägter als unter einer KK.

Parameter des oxidativen Stresses (DCF, MDA) sowie der Inflammation (hsCRP, TNF-alpha, Interleukin-6) wurden unter einer EK deutlicher vermindert als unter einer KK. Auch Veränderungen beim E-Selektin (Adhäsionsmolekül), den freien Fettsäuren und dem Adiponektin (Adipokin) waren unter hoher Eiweißzufuhr als metabolisch vorteilhaft einzuschätzen.

Kitabchi AE et al. Effects of high-protein versus high-carbohydrate diets on markers of beta-cell function, oxidative stress, lipid peroxidation, proinflammatory cytokines, and adipokines in obese, premenopausal women without diabetes. Diabetes Care 2013;36:1919-1925

Kommentar: Vier große Studien in den letzten Jahren zeigten, dass eine Gewichtsabnahme primär vom Ausmaß der Energierestriktion und kaum von der Relation der Makronährstoffkomponenten Eiweiß, Fett und Kohlenhydrate abhängt. Aus dieser
Erkenntnis ist jedoch nicht zu schließen, dass das Verhältnis der Makronährstoffe keine gesundheitliche Bedeutung hat, schließlich ist die Gewichtsabnahme nur eine Größe, wenn auch eine wichtige.

Die vorliegende Studie ist die erste, die neben einer Änderung von Gewicht, Körperzusammensetzung und klassischen Risikofaktoren weitere Faktoren wie Inflammation, oxidativen Stress und Betazellfunktion einschließt, um das metabolische Ausmaß dieser beiden Kostformen besser abschätzen zu können. Die Erkenntnis ist eindeutig: "The higher ratio of protein to carbohydrate has significant positive effects".

Soll man nun generell adipösen Frauen (bei Männern liegen keine vergleichbaren Daten vor) eine eiweißreiche Kost zur Gewichtsabnahme empfehlen? Die Fachgesellschaften zögern immer noch mit dieser Empfehlung, wenngleich die Veränderung von vielen Stoffwechselgrößen seit Jahren für eine eiweißreiche Ernährung spricht. Eine eiweißreiche Kost zur Gewichtsreduktion wird voraussichtlich erst dann grundsätzlich empfohlen, wenn Endpunktdaten vorliegen, was bis jetzt nicht der Fall ist.

Bedenken bestehen bei Diabetikern, aber zum Teil auch bei Nichtdiabetikern, ob unter einer eiweißreichen Kost eine Verschlechterung der Nierenfunktion stattfindet. In der vorliegenden Studie wurde auch Urin gesammelt und dieser hinsichtlich Mikroalbuminurie, Kreatinin-Clearance und Harnstoff/ Stickstoff untersucht. Leider wurden die Daten in der Publikation nicht dargestellt, auch nicht diskutiert.

Prof. Dr. med. Alfred Wirth, Bad Rothenfelde

\section{Vorhofflimmern kann kognitiven Abbau fördern}

\author{
Bei älteren Menschen mit neu auftretendem Vorhofflimmern können \\ kognitive Leistungsminderungen früher auftreten als bei altersgleichen \\ Personen ohne Vorhofflimmern, auch wenn kein Schlaganfall vorliegt.
}

\footnotetext{
n der Längsschnittstudie wurden $\mathrm{Da}$ ten von 5150 Teilnehmern der Cardiovascular Health Study ausgewertet, die bei Studienbeginn in den Jahren 19891993 mindestens 65 Jahre alt waren und kein Vorhofflimmern und keinen
}

Schlaganfall hatten. In den folgenden Jahren wurde bei allen Studienteilnehmern erstmalig auftretendes Vorhofflimmern anhand von Krankenhausberichten und jährlichen EKG-Untersuchungen erfasst. Die kognitive Leis- tungsfähigkeit wurde während der Nachbeobachtungszeit regelmäßig bis zu neunmal pro Jahr mit dem modifizierten Mini Mental Status Test (mMMST) erhoben und mit einem Punktwert zwischen 0 und 100 quantifiziert, wobei höhere Punktwerte eine bessere Leistungsfähigkeit anzeigen. Personen, bei denen während des Follow ups ein Schlaganfall auftrat, wurden ab diesem Zeitpunkt von der Studie ausgeschlossen.

Während der durchschnittlich sieben Jahre dauernden Nachbeobachtungszeit 